\title{
Análisis del Registro Arqueofaunístico de Grupos Cazadores-Recolectores del Holoceno Tardío de la Llanura Aluvial del Paraná Medio (Sitio Los Bananos, Departamento Goya, Corrientes, Argentina)
}

\author{
Late Holocene Hunter-Gatherers from Middle Paraná Flood Plain: Los Bananos Site \\ Zooarchaeological Evidence (Goya, Corrientes, Argentina)
}

María Carolina Barboza ${ }^{i}$ Maite Martín ${ }^{\text {ii }}$

\section{RESUMEN}

En este trabajo se presentan los resultados del estudio de los restos arqueofaunísticos procedentes del Sitio Los Bananos (Goya, Corrientes, Argentina). Los mismos evidencian la ocupación por parte de grupos cazadores-recolectores-pescadores en la llanura aluvial del Paraná medio durante el Holoceno tardío ( $1000 \pm 20$ años AP). Se realizó la estimación de la composición anatómica y taxonómica de los conjuntos. Se examinaron diferentes aspectos tafonómicos y se evaluaron las modificaciones antrópicas. Los resultados indican que los taxa que habrían sido más explotados por estos grupos están representados principalmente por aquellos característicos de los ambientes fluviales de la zona.

Palabras clave: Sitio Los Bananos, Zooarqueología, Llanura Aluvial, Paraná Medio, Holoceno Tardío.

\section{ABSTRACT}

This paper presents zooarchaeological analysis of Los Bananos site remains (Goya, Corrientes,Argentine).This provides new information about hunter-gatherer-fishers occupation of Middle Paraná alluvial plain during the late Holocene (1000 220 AP). Animal remains were identified with regard to anatomy and taxonomy. The focus of the analysis was to differentiate anthropic modification and the action of other agents or taphonomic processes. As a result of the analysis, the importance of fluvial resources was infered.

Key words: Los Bananos Site, Zooarchaeology, Middle Parana,Alluvial Plain, Late Holocene

\footnotetext{
${ }^{i}$ MCONICET - Centro de Estudios Interdisciplinarios en Antropología, FHumyAr, Universidad Nacional de Rosario. Alvear 452 Dpto. 5 (2000) Rosario, Santa Fe, Argentina. Correo-e: mcbarboza@yahoo.com

ii Centro de Estudios Interdisciplinarios en Antropología, FHumyAr, Universidad Nacional de Rosario. 1ro. de mayo 1767 Piso 5 Dpto. c (2000) Rosario, Santa Fe, Argentina. Correo-e: maite_martin6@ hotmail.com
}

Recibido: 05-12-2012 - Revisado: 02-05-2013 - Aceptado: 23-11-2013 


\section{INTRODUCCIÓN}

En este trabajo se presentan los resultados del estudio del material óseo faunístico procedente del sitio Los Bananos, localizado en la margen izquierda de la llanura aluvial del Paraná Medio (Departamento Goya, Corrientes, Argentina, Figura I). La misma pertenece a la eco-región Delta e Islas del Paraná (sensu Burkart et al. 1999) y se caracteriza por un alto grado de biodiversidad (Bó 2005). Entre los distintos ambientes de la llanura aluvial, el sitio Los Bananos se emplaza en un albardón ubicado en el borde frontal de la terraza baja. El mismo se encuentra sujeto a la dinámica del riacho colindante, muy cambiante como consecuencia propia del río Paraná y sus tributarios.

El sitio posee una topografía relativamente uniforme. El material arqueológico se presenta de forma discontinua, estando la mayor concentración y variedad en el sector más elevado de la superficie que abarca el sitio (Barboza y Píccoli en prensa). Se excavaron sistemáticamente un total de II cuadrículas de $|x| \mathrm{m}$ (volumen excavado hasta el momento: $8,7 \mathrm{~m}^{3}$ ). En una cuadrícula se excavó hasta alcanzar el nivel estéril arqueológicamente (ca. 145 $\mathrm{cm}$ de profundidad desde la superficie), las restantes continúan siendo excavadas. $\mathrm{Si}$ bien el material faunístico se distribuye a lo largo de toda la secuencia, el trabajo se centra en el análisis macroscópico de los restos analizados hasta el momento procedentes de todas las cuadrículas. En este sentido, se busca establecer una tendencia preliminar general y promediada del registro arqueofaunístico. Las diferencias encontradas entre las ocupaciones serán objeto de análisis en un trabajo futuro.

Se poseen tres fechados radiocarbónicos obtenidos a partir del análisis de dos muestras procedentes del primer nivel $(11-16 \mathrm{~cm}$, aproximadamente) y una del segundo $(16-2 \mid \mathrm{cm}$ aproximadamente). Los resultados obtenidos para las muestras procedentes del primer nivel son $1020 \pm$ 100 años AP (AA89916, cerámica) y $1020 \pm 90$ años AP (AA89917, cerámica), y para el segundo $488 \pm 42$ años AP (AA97852, óseo faunístico). Las diferencias observadas entre los resultados arrojados por las muestras cerámicas y óseas, pueden deberse a que el carbón datado derive de una materia de origen natural presente en la arcilla, o de un temperante no contemporáneo al proceso de manufactura (véase
Bonsall et al. 2002). Futuros análisis radiométricos y un control independiente para los fechados sobre material cerámico brindarán un panorama más concluyente (Barboza y Píccoli en prensa).

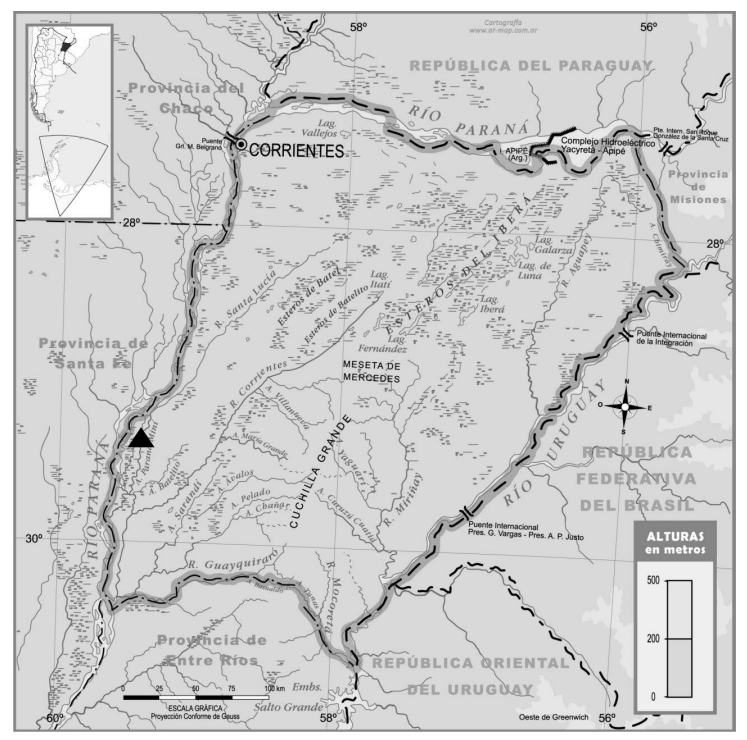

Figura 1: Ubicación del Sitio arqueológico Los Bananos.

Figure 1: Los Bananos site location

\section{MATERIALY MÉTODO}

Los restos faunísticos fueron asignados a distintos niveles taxonómicos. Aquellos que no pudieron ser asignados a una categoría fueron clasificados como indeterminados, contabilizados y separados de acuerdo estuviesen quemados o no. Las variables tafonómicas fueron estudiadas macroscópicamente con eventual auxilio de lupa 20x, excluyendo los indeterminados. Se registró la presencia de meteorización (Behrensmeyer 1978), abrasión sedimentaria, marcas de raíces, carnívoros y roedores (Binford I 98I, Fisher 1995). Los criterios utilizados para la identificación macroscópica de depósitos químicos son, por un lado, la presencia de concreciones externas de morfología diversa que se adhieren a la superficie cortical. Estas constituyen concreciones de ciertas sustancias químicas que por fenómenos de disolución y precipitación por repetidos humedecimientos y movimientos de agua, seguidos por desecación, terminan consolidándose (Conti 2000). Por otro lado, manchas negras aisladas 
o agrupadas, características de la precipitación de óxido de manganeso. Para el relevamiento de esta última variable, se utilizaron 4 categorías definidas en relación al porcentaje presente en la superficie: I (0-25\%), 2 (26- 50\%), 3 (5I-75\%) y 4 (76-100\%). Se relevaron evidencias de procesamiento de los restos óseos a fin de evaluar la acción antrópica sobre el conjunto faunístico. Se registró la presencia de alteración térmica a partir de la coloración de la superficie cortical; huellas de corte y fracturas antrópicas, producidas cuando el hueso se encontraba en estado fresco (Binford 198I, Fisher 1995, Johnson 1985, Shipman 1981).

\section{RESULTADOS}

La muestra total asciende a 239 I fragmentos, correspondientes a especímenes óseos, piezas dentales y restos malacológicos (n:3). Del total, 1809 (76\%) especímenes pudieron ser determinados, se encontraron 31 taxa representados, siendo once determinados a nivel de género y especie (Tabla I). En la mayoría de los casos las piezas dentales aisladas permitieron la determinación a nivel de especie. Esto es así especialmente en la muestra de peces, los que fueron clasificados en dos grandes grupos taxonómicos. El tamaño promedio de los especímenes del conjunto es $\leq 8.19 \mathrm{~mm}$. Quinientos ochenta y dos fragmentos (24\%) fueron clasificados como indeterminados, de los que 20I (35\%) presentan alteración térmica.

\begin{tabular}{|c|c|c|c|c|}
\hline Determinación taxonómica & NISP & $\%$ & MNE & $\%$ \\
\hline Aves & 41 & $2 \%$ & 37 & $2 \%$ \\
\hline Anseriforme & I & $0 \%$ & I & $0 \%$ \\
\hline Caimán sp. & 74 & $4 \%$ & 73 & $4 \%$ \\
\hline Squamata & I & $0 \%$ & I & $0 \%$ \\
\hline Teiidae & 4 & $0 \%$ & 4 & $0 \%$ \\
\hline Peces & 628 & $35 \%$ & 626 & $37 \%$ \\
\hline Siluriformes & 434 & $24 \%$ & 420 & $25 \%$ \\
\hline Characiformes & 104 & $6 \%$ & 97 & $6 \%$ \\
\hline Mammalia & 257 & $14 \%$ & 221 & $13 \%$ \\
\hline Cervidae & 27 & $1 \%$ & 14 & $1 \%$ \\
\hline Blastocerus dichotomus & 7 & $0 \%$ & 5 & $0 \%$ \\
\hline Rodentia & 83 & $5 \%$ & 68 & $4 \%$ \\
\hline Caviidae & 2 & $0 \%$ & 2 & $0 \%$ \\
\hline Ctenomys sp. & 16 & $1 \%$ & 16 & $1 \%$ \\
\hline Hydrochoerus hydrochaeris & 9 & $0 \%$ & 7 & $0 \%$ \\
\hline Lagomorpho & 8 & $0 \%$ & 3 & $0 \%$ \\
\hline Myocastor coypus & 68 & $4 \%$ & 53 & $3 \%$ \\
\hline Dasypodidae & 23 & $1 \%$ & 23 & $1 \%$ \\
\hline Chaetophractus villosus & 2 & $0 \%$ & 2 & $0 \%$ \\
\hline Dasypus hybridus & 15 & $1 \%$ & 15 & $1 \%$ \\
\hline Anura & 2 & $0 \%$ & 2 & $0 \%$ \\
\hline Bivalvia & 3 & $0 \%$ & 3 & $0 \%$ \\
\hline Total & 1809 & & 1690 & \\
\hline
\end{tabular}

Tabla 1: NISP y MNE representados para las distintas categorías taxonómicas identificadas.

Table 1: NISP and MNE by taxonomic categories 
La muestra se encuentra casi en su totalidad fragmentada $(97 \%, \mathrm{n}: \mid 76 \mathrm{I})$. El material analizado presenta una baja frecuencia de elementos enteros (3\%, n: 48), estos últimos representados por piezas dentales aisladas de peces, así como también vértebras, huesos del tarso y metapodios de distintas especies. En relación a la longitud de los especímenes recuperados, los de mayor tamaño corresponden a mamíferos (especialmente, huesos largos de cérvidos y roedores).

Las restantes variables que dan cuenta de los procesos naturales presentan una frecuencia relativamente baja (ver Tabla 2). En este sentido, se destaca la abrasión ( $\mathrm{n}: 315,17 \%)$ y la tinción por óxido de manganeso, aunque en la mayoría de los casos la porción del hueso afectada es reducida. El accionar de raíces y las marcas de roedores se registraron en un $2 \%$ de la muestra, mientras que las marcas de carnívoros en el $1 \%$. La meteorización se presenta en el $1 \%$ del conjunto, prevaleciendo los primeros estadíos. La presencia de las dos primeras variables $y$, en menor frecuencia de depósitos químicos (I\%), se encuentra asociada a ambientes húmedos (Alcaraz 2012, Conti 2000, Gutiérrez 2004, Martín et al. 2004), como en el que se encuentra emplazado el sitio y podría estar relacionada con las fluctuaciones de la napa freática y/o a los avances estacionales del riacho colindante. Sin embargo, el último factor mencionado se ha registrado solo excepcionalmente en el sector estudiado por tratarse de una porción elevada del terreno. La baja frecuencia de especímenes meteorizados así como también afectados por la acción de roedores y/o carnívoros, estaría indicando un enterramiento rápido y la escasa perturbación posdepósito.

Entre las modificaciones antrópicas observadas se destaca la alteración térmica $(20 \%$, n: 359). Ésta presenta mayor frecuencia en huesos largos de mamíferos, especialmente en cérvidos, osteodermos de Caiman sp., placas de dasypódidos, huesos largos y costillas de aves, vértebras de Teiidae y distintas porciones esqueletarias de peces $y$ roedores.

\begin{tabular}{|c|c|c|}
\hline Variable & Frecuencia absoluta & $\%$ \\
\hline \multicolumn{3}{|l|}{ Óxido de manganeso } \\
\hline Estadío 0 & 1554 & 0.859 \\
\hline Estadío I & 105 & 0.058 \\
\hline Estadío 2 & 35 & 0.019 \\
\hline Estadío 3 & 7 & 0.004 \\
\hline Estadío 4 & 5 & 0.003 \\
\hline \multicolumn{3}{|l|}{ Meteorización } \\
\hline Estadío 0 & 1793 & 0.991 \\
\hline Estadío I & 8 & 0.004 \\
\hline Estadío 2 & 3 & 0.002 \\
\hline Estadío 3 & 2 & 0.001 \\
\hline Estadío 4 & 0 & \\
\hline Estadío 5 & 0 & \\
\hline Abrasión & 315 & 0.174 \\
\hline Raíces & 33 & 0.018 \\
\hline Depósitos químicos & 10 & 0.006 \\
\hline Roedores & 29 & 0.016 \\
\hline Carnívoros & 14 & 0.008 \\
\hline
\end{tabular}

Tabla 2: NISP de las variables tafonómicas identificadas en los restos óseos.

Table 2: NISP by taphonomic variables.

Se registraron huellas de corte en el $5 \%$ del NISP total (n:87), principalmente en mamíferos (n:43), roedores ( $\mathrm{n}: \mathrm{I})$, M. coypus ( $\mathrm{n}: 10)$, H. hydrochaeris $(n: I)$, cérvidos $(n: 9)$, peces $(n: I)$, siluriformes $(n: 7)$, Caiman sp. (n:7), aves (n:7) y dasypódidos (n:I). Tampoco se registró una alta frecuencia de fracturas efectuadas sobre hueso en estado fresco $(1 \%, \mathrm{n}: \mid 8)$; las identificadas fueron realizadas sobre huesos largos de mamíferos, cérvidos y espinas de peces siluriformes. La baja frecuencia de huellas de corte $y$ fracturas podría ser atribuida al estado fragmentario de la muestra y/o al tamaño de los taxa presentes. Nada es posible decir respecto a la tecnología lítica utilizada por los grupos que ocuparon este sector, dado que hasta el momento solo se han registrado desechos de talla (microlascas) en el sitio (Píccoli y Gavilán 2013). 


\section{CONSIDERACIONES FINALES}

Este trabajo constituye una caracterización general de la muestra de materiales óseos faunísticos recuperados en el Sitio Los Bananos analizados hasta el momento. Sobre la base de este análisis se puede postular que el conjunto recuperado habría ingresado preferentemente al sitio por acción antrópica. Esto no solo podría sostenerse para el caso de los especímenes que presentan modificaciones culturales, sino también en general por la gran diversidad de taxa representados (correspondientes a animales de pequeño y mediano porte propios del ambiente en el que se emplaza el sitio).

Por otro lado, la representación diferencial de elementos enteros vs. fragmentados en el conjunto podría estar relacionada con las características óseas que presentan los taxa representados, y/o con la intervención antrópica vinculada con actividades de procesamiento. Otra hipótesis plausible, tomando en cuenta la dimensión de los especímenes, podría estar relacionada con el tipo de matriz sedimentaria en la que se encuentran los restos, junto con la presión propia de la ocupación actual del sector. Asimismo, si se considera que los taxa de mayor tamaño y menor frecuencia en la muestra son los que más presencia de huellas antrópicas evidencian, mientras que en aquellos de menor porte y mayor representación esta variable disminuye, cabe pensar que la presencia de huellas se encuentra subrepresentada. Como futura línea de análisis se considera que la estimación del número mínimo de individuos por taxa posibilitará entender de qué forma fueron aprovechados los recursos por parte de los grupos que habitaron la zona en el pasado.

\section{AGRADECIMIENTOS:}

Las autoras agradecen a los alumnos que participaron de los trabajos de campo efectuados en 2009-2012; a la Dra. Lorena L'Heureux; los Lic. Carolina Píccoli, Carlos Ceruti, David Ávila, Carolina Gabrielloni, Jimena Cornaglia Fernández y a la Prof. Andino. A Martín Stochero. A la Secretaría de Producción, Empleo y Desarrollo Sustentable, Municipalidad de Goya, por el apoyo logístico brindado durante los mencionados trabajos. A la Dirección de Flora y Fauna, Delegación Goya, por el suministro de material comparativo. A la comunidad escolar de la Escuela $N^{\circ} 276$ del Paraje Stella Maris y a la comunidad del paraje en general. Este trabajo se desarrolla en el marco del proyecto "Estudios sobre las construcciones sociales del paisaje en la Llanura Aluvial del Paraná Medio (Departamento Goya, Provincia de Corrientes)" (CyT, FHumyAr, UNR y PIP-CONICET N ${ }^{\circ}$ 0I39/10). La versión inicial de este trabajo fue mejorada a partir de los comentarios efectuados por los revisores, cuyas críticas y aportes también agradecemos. Los errores u omisiones son de nuestra exclusiva responsabilidad.

\section{BIBLIOGRAFÍA}

Alcaraz, A. 2012. Análisis zooarqueológico y tafonómico de restos óseos de pequeños vertebrados de sitios del curso inferior del río Colorado (Pcia. de Buenos Aires) durante el Holoceno tardío:Aportes para el conocimiento de la subsistencia $t$ procesos de formación. Tesis de Licenciatura. Facultad de Ciencias Sociales, Universidad Nacional del Centro de la Provincia de Buenos Aires.

Barboza, C. y C. Píccoli 2012."Ocupaciones Humanas en la Llanura Aluvial del Paraná Medio durante el Holoceno Tardío. El registro arqueológico del Sitio Los Bananos (Goya, Corrientes, Argentina)". Revista Anuario de Arqueología. En prensa.

Behrensmeyer, K. 1978. "Taphonomic and Ecologic Information from Bone Weathering”. Paleobiology 4: I50-I62.

Binford, L. 1981. Bones: Ancient Men and Modern Myths. Academic Press, New York.

Bó, R. 2005. "Situación ambiental en la ecorregión Delta e Islas del Paraná”. En La Situación Ambiental Argentina, editado por A. Brown, O. Martinez, U. Ortiz, M.Acerbi, y J. Corcuera, pp. I3 | - I 43. Fundación Vida Silvestre Argentina, Buenos Aires.

Bonsall, C.; Cook, G.; Manson, J. y D. Sanderson 2002."Direct dating of Neolithic pottery: progress and prospects". Documenta Praehistorica XXIX: 47-59.

Burkart, R.; Bárbaro, R.; Sánchez, R. y D. Gómez 1999. Ecoregiones de la Argentina. Administración de Parques Nacionales. Programa de Desarrollo Institucional Ambiental. Secretaría de Recursos Naturales y Desarrollo Sustentable.

Conti, M. 2000. Principios de edafología. Con énfasis en suelos argentinos. Ed. Fac. de Agronomía, Buenos Aires.

Fisher, J. 1995. "Bone Surface Modifications in Zooarchaeology".Journal of Archaeological Method andTheory 2(I):7-68.

Gutiérrez, M. 2004. Análisis tafonómico en el Área Interserrana (Provincia de Buenos Aires). Tesis Doctoral. Facultad de Ciencias Naturales y Museo, Universidad Nacional de La Plata.

Johnson, E. 1985. "Current developments in bone technology". En Advances in Archaelogical Method and Theory 8, editado por M. Schiffer, pp. 157-235. Academic Press, Orlando.

Martin, F. Barberena , R. y R. Guichón. 2004. "Erosión y huesos humanos. El caso de la localidad chorrillos, tierra del fuego”. Magallania 32:125-142.

Píccoli, C. y M. Gavilán. 2013. "Análisis del material lítico procedentes de la Llanura Aluvial del Paraná Medio (Goya, Corrientes)". Libro de Actas de las VI Jornadas de Ciencia y Técnica, pp. 438-442. Universidad Nacional de Rosario, Rosario.

Shipman, P. 1981. "Applications of scanning electron microscopy to taphonomic problems". Annals of the New York Academy of Sciences 276: 357-386. 